\title{
SCALABLE MACHINE LEARNING ALGORITHMS FOR A TWITTER FOLLOWEE RECOMMENDER SYSTEM
}

\author{
Sepideh Banihashemi \\ Jason Li \\ Abdolreza Abhari \\ Department of Computer Science \\ Ryerson University \\ 245 Church Street, ENG-234 \\ Toronto, ON, Canada \\ \{sepideh.banihashemi,jcli,aabhari\}@ryerson.ca
}

\begin{abstract}
Recently, machine learning (ML) algorithms have been employed in social networking recommender systems. In this paper, a Twitter recommender system is simulated by a multi-agent system that can be used to provide the users with a list of useful recommendations, specifically a list of users (i.e., followees) that a user is interested in following. The simulator is used to test the scalability of a machine learning algorithm (i.e., Neural Network, Multilayer Perceptron) for data analysis with parallel implementation on multi-node distributed systems. The distributed environment is simulated by a multi-agent modeling. The initial parameters that should be set up on the simulator include the number of nodes, the algorithm employed in the simulated recommender system, and the actual followees and followers information. The experimental results were obtained on three distinct datasets for evaluating the accuracy and the execution time of a simulated recommender system when testing the ML algorithm in different scenarios.
\end{abstract}

Keywords: Simulating Recommender Systems, Scalable Machine Learning, Multilayer Perceptron.

\section{INTRODUCTION}

Data analysis can be performed by the algorithms that extract information from data and produce a model which can be used in the future. These machine learning algorithms are grouped into supervised and unsupervised algorithms. In supervised machine learning algorithms, the data has classification labels. On the other hand, the input data in unsupervised machine learning algorithms are not labeled and thus, there is not any obvious information about the data (Leskovec, Rajaraman and Ullman 2014).

Multilayer Perceptron (MLP) is a neural network model which has got its inspiration from the human brain and it can be used for supervised learning. Each perceptron is a fundamental unit that contains input which is generated as an output of the other perceptron. Additionally, the weights connected to the inputs are applied to compute the output of the network (Alpaydin and Bach 2014).

In online social network services such as Twitter, the users are posting information in short messages which are called tweets. In such a network, a user that follows another user is considered as a follower while the other user would be considered as a followee. In addition, followees are behaving as the information sources 
as they are collecting a large number of followers who are the information seekers (Armentano, Godoy and Amandi 2012).

Recommender systems are applied to look for the users' preferences and create a list of recommendations for a specific user (Jannach, et al. 2010). In terms of Twitter, a recommender system can generate a list of users that another user might be interested in following.

A multi-agent simulator that is developed for simulation of the recommender system in our lab (Abhari 2014 ) is used for this work. A clustering algorithm (i.e., unsupervised learning) has been employed in the simulated recommender system and original results reported in ( $\mathrm{Li}$ and Abhari 2017) with comprehensive scalability test for both follower and followees recommendation with the results explained in (Li 2019). In this paper, we used a supervised machine learning algorithm (i.e., Multilayer Perceptron) and limited the recommender system only to recommend a person to follow (i.e., a followee). Similar to our previous works, we changed the machine learning algorithm to be able to work in a distributed environment for providing scalability and reported the performance in this paper.

Machine learning is a discipline which has developed in many areas and concentrates on prediction through experience, and it can be trained by instances of desired labels for each input. Optimizing the performance metric can be considered as the objective of machine learning algorithms. With the emergence of big data, machine learning algorithms are applied to solve the problem of making appropriate predictions and decisions from such datasets. Moreover, the scalable algorithms are necessary as they can learn from these data and perform mining of the vast amount of data. Machine learning methods can be categorized into supervised and unsupervised learning techniques. In supervised learning, the dataset is labeled and the objective is to make predictions based on the learning map. On the other hand, unsupervised learning analyzes the unlabeled data. In terms of the architecture, scalable machine learning systems are composed of structures which are made on parallel and distributed platforms (Jordan and Mitchell 2015).

In (Mamgain, et al. 2016), Twitter data related to three top colleges in India is collected including the user, time-zone, location, etc. The preprocessing method is done on the tweets to separate the user ideas from user information such as omitting the URLs, some punctuations in the tweets, consecutive repeated letters, and useless symbols. $75 \%$ of the data is applied for the training phase and the rest for the test phase of the machine learning algorithm (i.e., Artificial Neural Network, Multilayer Perceptron). The training data is transferred into the input layer and passed through the first and second hidden layers to generate the output and the activation function used on both hidden layers is Sigmoid function.

The Artificial Neural Network (ANN) is an effective algorithm for the classification task. Additionally, the Backpropagation Neural Network (BPNN) adjusts the weights of the neural network by utilizing an error backpropagation method and can be performed in parallel by applying MapReduce which spreads the iteration and computation of BPNN in parallel. BPNN distributes the data to mappers to classify and the outputs of the mappers will be grouped by the reducer to produce the classification rules ( $\mathrm{Liu}, \mathrm{Xu}$ and $\mathrm{Li}$ 2017).

Parallel implementation of Multilayer Perceptron can be developed as a MapReduce task. The training dataset is divided into several pieces and each part is assigned to a Map function with its connected weights. The Map task then calculates the dot product of each input vector with its associated weight and compares it with its label which is +1 or -1 . If the sign of the dot product and its label does not match then for each $\mathrm{x}_{\mathrm{i}}$ that is nonzero, a key-value pair $\left(\mathrm{i}, \eta \mathrm{yx}_{\mathrm{i}}\right)$ is made in which $\eta$ is the learning rate. The Reduce function adds all the $\eta x_{i}$ for the related $i$ and finally, the produced sum will be added to the $\mathrm{w}_{\mathrm{i}}$. If there are any changes to the $\mathrm{w}$, then the training phase will be repeated by splitting the training set to different pieces (Leskovec, Rajaraman and Ullman 2014). The difference between this work and our work is that we don't simulate MapReduce as a parallel and distributed environment. Instead, we simulate any distributed system that can be built by Apache Spark servers or multi-node processors. 


\section{METHODOLOGY}

Initially, real tweets were collected in order to model the tweets. While gathering the data from the Twitter API, various information will be collected and not all of them are useful for conducting the experiment. The tweets which are going to be fed into the simulator are formatted as the table 1 below.

Table 1: Example format of the data gathered and to be used in simulator.

\begin{tabular}{|c|c|c|c|c|c|}
\hline FolloweeName & TweetID & TweetDate & UserID & FollowerName & TweetText \\
\hline UserA & 722196833803268620 & $\begin{array}{c}2016-03- \\
22\end{array}$ & 123456 & UserB & $\begin{array}{c}\text { RT @UserA: Do } \\
\text { not stop retweeting } \\
\text { this } \\
\text { this }\end{array}$ \\
& & & & & https:/t.co/VnU1e3 \\
\hline
\end{tabular}

As shown in the table 1 above, the data from Twitter are formatted in the order of followee name, the ID of the tweet, the date and time the tweet was posted, the user ID of the tweet, the user name of the user that posted the tweet, and the text of the tweet. The tweets of a Twitter follower were collected before following their respective followee and this can be used to verify the accuracy as it is known who the Twitter user is following.

\subsection{Preprocessing the Data}

Tf-idf is a common method to verify the values of weights for an input vector which can be considered as the document, $d$, and contains all the tweets of a specific user. The term tf-idf refers to term frequency, inverse document frequency. The term frequency specifies the number of the occurrence of a term, $t$, in a document, $d$, by considering that the words in a tweet are the terms. The document frequency, $d f$, specifies the number of documents in which a term occurs (Manning, Raghavan and Schütze 2008). The tf-idf method is applied to all the documents, where each document is a user's tweets combined into a single document.

\section{IMPLEMENTATION AND RESULTS}

\subsection{Datasets}

In this experiment, 3 different datasets were used. The RU3_Set is a dataset which contains 3 followees and 3 followers for each followee. The MDS_Set dataset has $\overline{4}$ followees and 5 followers for each followee. The LDS_Set dataset includes 4 followees and 9 followers for each followee. After preprocessing the datasets, the number of unique words is determined. Table 2 illustrates each dataset along with its relevant information.

Table 2:Datasets used for the simulations.

\begin{tabular}{|c|c|c|c|c|}
\hline Dataset & $\begin{array}{c}\text { Number of } \\
\text { Tweets }\end{array}$ & $\begin{array}{c}\text { Number of } \\
\text { Followers }\end{array}$ & $\begin{array}{c}\text { Number of } \\
\text { Followees }\end{array}$ & $\begin{array}{c}\text { Number of } \\
\text { Unique Words }\end{array}$ \\
\hline RU3_Set & 14,265 & 9 & 3 & 23,676 \\
\hline MDS_Set & 57,763 & 20 & 4 & 53,905 \\
\hline LDS_Set & 94,547 & 36 & 4 & 86,658 \\
\hline
\end{tabular}




\subsection{The Testing Environment}

The simulations were run for the RU3_Set, MDS_Set and LDS_Set datasets on a $4.0 \mathrm{GHz}$ computer with 16 GB RAM and Windows 7 OS.

Additionally, Java was installed on the machine to enable it to run the simulator which was developed in Java. The performance and timing results were achieved from running the simulator on this machine.

\subsection{Implementation}

The multi-agent modeling is utilized for simulating a distributed environment and Twitter users. Multiagent models are effective tools for simulation of distributed computing and recommender systems. The multi-agent model for conducting the experiment includes the controller agent, organizing agent, user agent(s), recommender agent(s), starter agent, and data crawler agent. The experiment is utilizing each individual agent.

The controller agent controls generating all the various agents and the flow of the simulation by managing the time of data collection and launching a simulation for the purpose of scalability performance evaluation. The controller agent provides the user name of the followee and the number of the followers to the data crawler agent. Another task of the controller agent is to specify the way tweets can be distributed between the recommender agents.

The organizing agent controls if the recommender agent has the correct number of user agents. Once a user agent is built, it informs the organizing agent that it is ready. The recommender agent controls the timing of the algorithms when they are running, and each recommender agent is in charge of the recommendation list that a user agent asks for. If there are several recommender agents, each recommender agent sends the recommendation list to the organizing agent to combine all into one sole list.

The starter agent is responsible for starting each simulation iteration when all the other agents are ready. It is also the agent that initiates the recommendation algorithm. The data crawler agent collects data using the Twitter API for the experiments. A user agent represents a Twitter user in the dataset that sends tweets and queries the simulated recommender system for a recommendation of users to follow.

In this experiment, there can be 1, 2, 4 or 8 recommender agents each representing a single node. This node simulates a processing component in a distributed system. In the case of 1 single node, the MLP machine learning method is applied as a classifier which trains from $70 \%$ of the whole dataset for the training phase and generalizes the model on the $30 \%$ of the remaining dataset for the test phase.

On the other hand, 2 or more recommender agents can perform MLP on each node separately in parallel by splitting the whole dataset as evenly as possible. Afterward, each recommender agent divides its portion of data to $70 \%$ for the training and $30 \%$ for the test phase. First, the weighted sum is calculated in each hidden neuron and passed through the activation function to transfer the output to the output neurons in the output layer. The activation function utilized in the hidden neurons is Tangent hyperbolic and the activation function used in the output neurons is the Softmax function. Then, the weighted sum of the hidden neurons after being applied by the activation function is transferred to output neurons to produce the output. The backpropagation method is applied to adjust the connected weights of MLP and finally, the connected weights for each neuron in all the recommender agents are averaged. The extracted model will be generalized on the test dataset to obtain the accuracy of each recommender agent along with the execution timing. The total accuracy can then be calculated by adding up the correctly classified instances divided by the sum of the total number of instances in each agent.

The number of the neurons in the hidden layer is 10 as defined by the rule of thumb technique in which the number of hidden neurons should be in the range between the number of the input neurons and the number of the neurons in the output layer (Panchal and Panchal 2014). The number of the neurons in the input layer is 86658 if the dataset LDS_Set is used, 53905 if the MDS_Set is utilized and 23676 if the RU3_Set is applied. The Learning rate is 0.1 and the Max error rate is 0.01 . These previous values are the $\overline{\text { default }}$ 
parameters, initialized by the Neuroph library implementation of the Multilayer Perceptron (Neuroph Java Neural Network Framework 2017).

Figure 1 shows the screenshot of the running simulator when the test phase has been concluded, set on 8 nodes, MLP algorithm, and the user getting the recommendation.

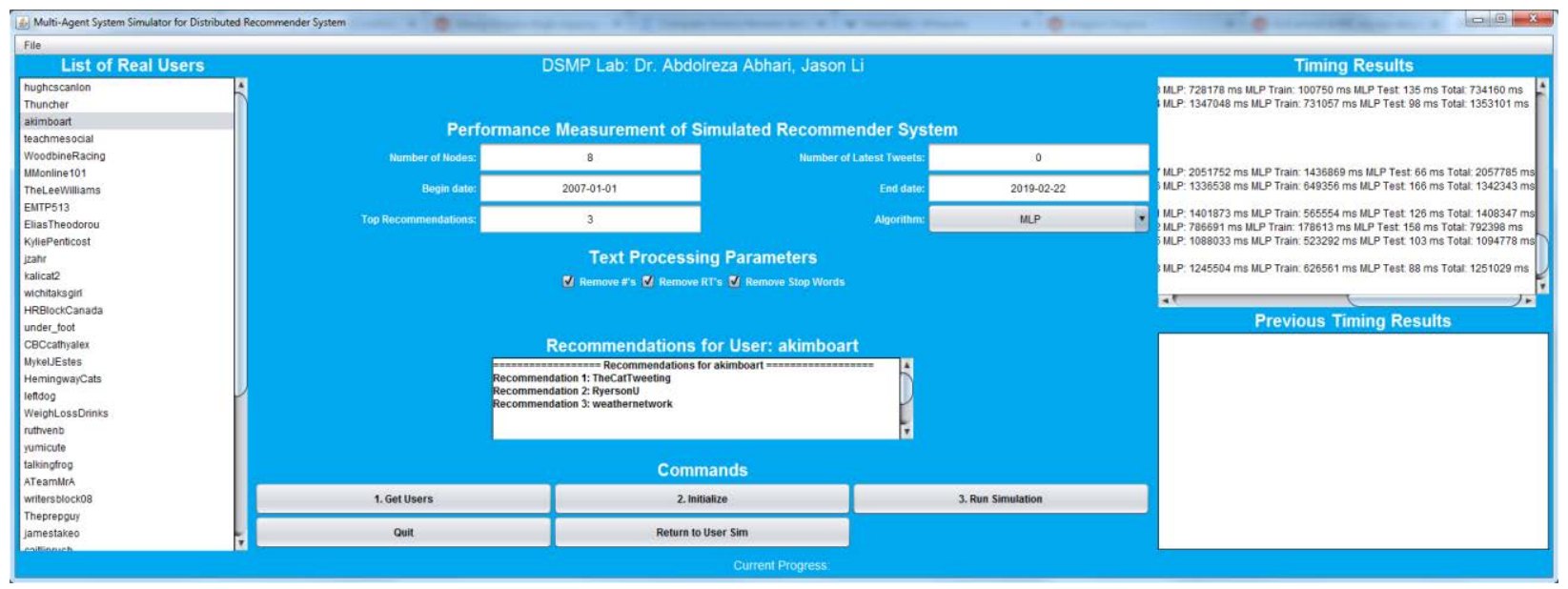

Figure 1:Screenshot of the simulator used in the experiments.

\subsection{Results}

In order to obtain the performance results in this experiment, the simulator was run by initially setting up the parameters such as the number of nodes, the developed algorithm, and the tweets of actual followees and followers. The Training-Time specifies the running time it takes to train the Multilayer Perceptron and the Test-Time defines the running time to feed the test dataset to MLP. The MLP-Timing consists of the training-time, test-time and the communication cost of each node, which is the time waiting for the weights of the MLP from other nodes to be averaged. The performance improvement is calculated by taking the ratio of the timing result as the number of $\mathrm{X}$ nodes increases compared to 1 node.

$$
\text { Performance Improvement }=\frac{\text { Timing of } 1 \text { Node-Timing of X Node }}{\text { Timing of } 1 \text { Node }} * 100 \text {. }
$$

The following table 3 shows the results of the simulation experiment running MLP on 1 node as well as 2 and 4 nodes on RU3_Set dataset. The results show that adding more nodes reduces timing of training and detection of the machine learning algorithms while variating the accuracy in the range of $10 \%$ to $20 \%$. Additionally, the accuracy decreases around $10 \%$ when adding more nodes to the simulator. The experiment was not applicable for 8 nodes with this dataset due to the number of users since after distributing the users to each node equally, some nodes do not get enough number of users for the training and test phases in an individual node. Moreover, running the RU3_Set dataset with 2 nodes has a time decrease of $44.79 \%$ and running it with 4 nodes has got a time decrease of $54.62 \%$. 
Table 3:Results of Accuracy and Timing for RU3_Set.

\begin{tabular}{|c|c|c|c|c|}
\hline Number of Nodes & Accuracy & Training-Time & MLP-Timing & $\begin{array}{c}\text { Performance } \\
\text { Improvement }\end{array}$ \\
\hline 1 & $55.56 \%$ & $356,109 \mathrm{~ms}$ & $407,225 \mathrm{~ms}$ & \\
\hline 2 & $44.44 \%$ & $179,033 \mathrm{~ms}$ & $224,820 \mathrm{~ms}$ & $44.79 \%$ \\
\hline 4 & $33.33 \%$ & $139,427 \mathrm{~ms}$ & $184,763 \mathrm{~ms}$ & $54.62 \%$ \\
\hline
\end{tabular}

The table 4 below displays the results of the simulation experiment running MLP on 1, 2, 4, and 8 nodes on MDS Set dataset. The results show that adding more nodes decreases the timing of training. In other words, the results of the simulation for MDS Set dataset with 2 nodes has obtained a timing decrease of $35.33 \%$ and running it with 4 nodes has the timing decrease of $50.07 \%$. In addition, running this dataset with 8 nodes has got the timing decrease of $70.31 \%$.

Table 4:Results of Accuracy and Timing for MDS_Set.

\begin{tabular}{|c|c|c|c|c|}
\hline Number of Nodes & Accuracy & Training-Time & MLP-Timing & $\begin{array}{c}\text { Performance } \\
\text { Improvement }\end{array}$ \\
\hline 1 & $42.86 \%$ & $2,570,669 \mathrm{~ms}$ & $2,645,067 \mathrm{~ms}$ & \\
\hline 2 & $25 \%$ & $1,601,027 \mathrm{~ms}$ & $1,710,417 \mathrm{~ms}$ & $35.33 \%$ \\
\hline 4 & $10 \%$ & $1,145,386 \mathrm{~ms}$ & $1,320,616 \mathrm{~ms}$ & $50.07 \%$ \\
\hline 8 & $7 \%$ & $449,938 \mathrm{~ms}$ & $785,210 \mathrm{~ms}$ & $70.31 \%$ \\
\hline
\end{tabular}

Table 5 below presents the results of the simulation experiment running MLP on 1 node as well as 2, 4, and 8 nodes on LDS_Set dataset. The results of the simulation for LDS_Set dataset with 2 nodes has achieved the timing decrease of $82.04 \%$ and has got the timing decrease of $91.87 \%$ with 4 nodes. Moreover, the results of the simulation for this dataset with 8 nodes has obtained the timing decrease of $92.61 \%$. The accuracy of LDS_Set dataset has a difference of approximately $10 \%$ to $20 \%$ to the accuracy of running the simulator on 2 , and 4 nodes.

Table 5:Results of Accuracy and Timing for LDS_Set.

\begin{tabular}{|c|c|c|c|c|}
\hline Number of Nodes & Accuracy & Training-Time & MLP-Timing & $\begin{array}{c}\text { Performance } \\
\text { Improvement }\end{array}$ \\
\hline 1 & $41.67 \%$ & $22,595,837 \mathrm{~ms}$ & $22,762,730 \mathrm{~ms}$ & $82.04 \%$ \\
\hline 2 & $30.76 \%$ & $3,838,088 \mathrm{~ms}$ & $4,086,791 \mathrm{~ms}$ & $91.87 \%$ \\
\hline 4 & $20 \%$ & $1,530,656 \mathrm{~ms}$ & $1,849,889 \mathrm{~ms}$ & $92.61 \%$ \\
\hline 8 & $15.78 \%$ & $1,002,722 \mathrm{~ms}$ & $1,681,329 \mathrm{~ms}$ & \\
\hline
\end{tabular}

\section{CONCLUSION}

In this paper, the single node and multiple node environments are used to run recommender agents in parallel to evaluate the accuracy and timing of the implemented MLP algorithm for an actual Twitter followee recommender system. The results show that the MLP execution timing of each individual dataset on 1 node is more than the MLP execution timing on 2, 4, and 8 nodes. Additionally, the accuracy of 
LDS_Set dataset running on 1 node is deviated only by more than $10 \%$ compared to the accuracy of running the simulator with 2 nodes. For 2 node observation in all datasets, parallel recommender agents executing the MLP algorithm are able to reduce the timing with a minimum of $35 \%$ and with the cost of decreasing the accuracy of at least $10 \%$. The interesting observation is when the largest dataset, around $94 \mathrm{~K}$ tweets, is used (i.e., LDS_Set) the performance improvement of running time is more while the accuracy decrease has become less when adding more nodes. This result shows that the methodology proposed in this paper can make machine learning algorithm scalable for the Twitter social networking service when recommending for millions of tweets.

\section{REFERENCES}

Abhari, Abdolreza. 2014. Distributed Systems and Multimedia Processing Lab. Accessed 12 01, 2018. http://dsmp.ryerson.ca/.

Alpaydin, Ethem, and Francis Bach. 2014. Introduction to Machine Learning. Cambridge: MIT Press.

Armentano, Marcelo G, Daniela Godoy, and Analia Amandi. 2012. "Topology-Based Recommendation of Users in Micro-Blogging Communities." Journal of Computer Science and Technology 624-634.

Jannach, Dietmar, Markus Zanker, Alexander Felfernig, and Gerhard Friedrich. 2010. Recommender Systems : An Introduction. Cambridge: Cambridge University Press.

Jordan, Michael I, and T M Mitchell. 2015. "Machine learning: Trends, perspectives, and prospects." Science 255-260.

Leskovec, Jure, Anand Rajaraman, and Jeffrey D Ullman. 2014. Mining of Massive Datasets. Cambridge: Cambridge University Press.

Li, Jason. 2019. Generating Artificial Data for Scalability Test of a Followee Twitter Recommender System. Master's Thesis, Toronto: Ryerson University.

Li, Jason, and Abdolreza Abhari. 2017. "Generating Stochastic Data to Simulate a Twitter User." CNS '17 Proceedings of the 20th Communications \& Networking Symposium. Virginia Beach: Society for Computer Simulation International. 102-112.

Liu, Yang, Lixiong Xu, and Maozhen Li. 2017. "The Parallelization of Back Propagation Neural Network in MapReduce and Spark." International Journal of Parallel Programming 760-779.

Mamgain, Nehal, Ekta Mehta, Ankush Mittal, and Gaurav Bhatt. 2016. "Sentiment Analysis of Top Colleges in India Using Twitter Data ." 2016 International Conference on Computational Techniques in Information and Communication Technologies (ICCTICT). New Delhi: India. 525-530 .

Manning, Christopher D, Prabhakar Raghavan, and Hinrich Schütze. 2008. Introduction to Information Retrieval. Cambridge University Press: Cambridge.

2017. Neuroph Java Neural Network Framework. Accessed 12 01, 2018. http://neuroph.sourceforge.net/.

Panchal, Foram S, and Mahesh Panchal. 2014. "Review on Methods of Selecting Number of Hidden Nodes in Artificial Neural Network." International Journal of Computer Science and Mobile Computing 455464.

\section{AUTHOR BIOGRAPHIES}

SEPIDEH BANIHASHEMI is a Ph.D. student of Computer Science at Ryerson University. Her research interests lie in scalable machine learning algorithms, simulation, and object detection. Her email address is sepideh.banihashemi@ryerson.ca. 
JASON LI is a recently graduated Master's student of Computer Science at Ryerson University. His research interests lie in multi-agent systems, simulation, machine learning, and data science. His email address is jcli@ryerson.ca.

ABDOLREZA ABHARI is a Professor in the Department of Computer Science at Ryerson University and director of DSMP lab (http://dsmp.ryerson.ca). He holds a Ph.D. in Computer Science from Carleton University. His research interests include web social networks, data science, AI and agent systems, network simulation, and distributed systems. His email address is aabhari@ryerson.ca. 